\title{
Software Engineering Implementation Experience of Precision Navigating System
}

\author{
Na Zhang, Haifeng Jang \\ Beijing Institute of Aerospace Control Devices, Beijing, China
}

Email address:

zhangna_1122@163.com(Na Zhang),276873824@qq.com(Haifeng Jang)

\section{To cite this article:}

Na Zhang, Haifeng Jang. Software Engineering Implementation Experience of Precision Navigating System. Science Discovery.

Vol. 6, No. 1, 2018, pp. 47-51. doi: 10.11648/j.sd.20180601.18

Received: January 18, 2018; Accepted: April 16, 2018; Published: May 23, 2018

\begin{abstract}
The precision navigating system is widely used in the weapon system. With the development of the digital circuit technology,the software becomes the core of navigating system,all of the function are accomplished by embedded software.There is a high requirement for software quality、security、stability and reliability.In order to increase the success rate for software development and improve the quality of software, this paper summarizes the creative measures of software engineering implementation from software system design、software management、communication mechanism、 development process and reliability design in navigating system research process. The effectiveness of the measures is verified by research achievements.
\end{abstract}

Keywords: Precision Navigating System, Software Engineering, Software Quality

\section{精密导航系统软件工程化实施经验与启示}

\section{张娜, 姜海峰}

北京航天控制仪器研究所, 北京, 中国

\section{邮箱}

zhangna_1122@163.com (张娜), 276873824@qq.com(姜海峰)

摘要: 精密导航系统被广泛应用于武器型号中, 随着数字电路技术的不断发展和应用, 精密导航系统内的软件系统成 为实现整体能力的核心, 其对软件的质量、安全性、稳定性和可靠性等有着较高的要求, 为了提高软件开发的成功率 和软件产品的质量, 精确控制软件开发过程和软件开发进度,本文利用软件工程化的思维结合精密导航系统特点从软件 系统设计、软件管理、沟通机制、研制流程及可靠性设计5个方面总结了武器型号软件管理实施的创新性措施, 并通过 上述措施在精密导航系统软件研制过程的成功实施验证了措施的有效性。

关键词: 精密导航系统, 软件工程化, 软件质量

\section{1. 引言}

软件工程是一门工程科学, 主要讲述如何规范化的管 理和开发软件。尽管很多学者都各自给出了软件工程的定 义，但其基本思想一直保持一致：用工程化方法系统而合
理地规范软件产品的开发, 从而经济的获得可靠的、高效 运行的软件。

精密导航系统 (以下简称导航系统) 为武器系统提供 惯性测量基准, 并输出弹体视加速度和姿态角等信息。由 于其具有直接模拟导航坐标系, 计算简单; 能隔离载体角 运动, 系统精度高等特点, 被广泛应用于战略武器型号中, 
随着数字电路技术的不断发展和应用, 导航系统内的软件 成为实现系统能力的核心, 导航系统的重要功能均依托软 件实现。由于新型导航系统软件具有软、硬件技术状态新, 软件安全等级较高, 系统内配置项数目较多, 接口复杂, 需求迭代次数多, 测试验证复杂等难点, 为了有效而高质 量的进行精密导航系统的软件管理和研制工作, 根据软件 工程化的基本原理, 结合精密导航系统的研制特点, 在软 件研制工作过程中采取了多项创新型措施, 上述措施的有 效实施, 保证了软件研制的进度和质量, 同时也为后续型 号软件研制奠定了基础。

\section{2. 精密导航系统软件研制特点}

\section{1. 软、硬件技术状态新}

在导航系统方案设计阶段确定了硬件电路的设计准 则, 即数字化电路方案和国产化元器件的应用。因此对电 气系统方案进行了重新的设计, 且多个处理器采用了国产 化芯片, 同时软件系统方案也进行了较大的修改, 原有模 拟电路实现的核心功能, 如伺服回路控制、电源控制、温 度控制等均由软件实现，软、硬件设计中可借鉴沿用的技 术有限。新技术、新状态的大量应用与研制进度紧张形成 的矛盾成为在软件研制过程中的突出问题。

\section{2. 信息流复杂, 通讯链路长}

导航系统软件包含了十余个软件配置项, 各配置项内 部信息传输通道接口类型多, 信息类型丰富, 信息路径关 系复杂, 部分信息需通过三级以上的传输, 且每一级的传 输都需经过握手回复等环节, 使信息的梳理更加复杂, 同 时外部涉及与地面测发控、遥测等多个系统接口, 更增加 了软件接口的复杂性。

\section{3. 研制部门交叉}

导航系统软件的研发涉及多个研制单位及本单位多 个部门, 配置项之间的交互匹配性直接影响产品运行的正 确性和稳定性, 不同单位及部门之间进行并行研制并进行 交叉交付于验证, 给工程管理带来了新的特点。

\section{3. 精密导航系统软件工程化实践}

\section{1. 在系统设计早期开展软件系统设计工作}

对于工程设计来讲, 系统方案是对设计方法的确定。系 统方案的研究对于保证工程设计最终取得预定成果具有重 要的作用。嵌入式软件的开发方案与硬件架构具有密不可分 的联系, 其研制过程依赖硬件环境, 因此软件研制往往成为 整个系统研制的最后一个环节, 即确定了系统方案后形成单 个软件配置项的研制任务书才开始软件的研制工作。系统方 案的制定由总体及硬件设计人员完成, 这类方案仅考虑了总 体及电气功能的实现, 缺乏对软件系统拓扑结构的细致划分 及进一步优化, 同时也没有考虑整体的信息流 (包括数据流、 指令流及接口关系) 的整体设计, 对软件系统最终的可靠性 有着较大的影响, 而且这种影响是不可逆的。因此在导航系 统研制过程中, 软件设计人员在早期就介入到整体方案制定 中, 从软件专业的角度提出具体建议, 从而优化系统设计方 案, 并完成软件系统设计工作。

软件系统设计就是定义软件系统的静态结构和动态过 程, 其中静态结构包括确定软件系统的组成部分、软件拓扑 结构及物理连接关系、相互交换什么样的指令和数据（逻辑 连接关系）、这些指令和数据会激发接收方的什么响应。动 态过程包括通信的时序关系, 包括通信的先后顺序、超时时 间、等待时间、重发机制、拥塞管理, 也包括通信双方对接 口信息的处理过程和统一化要求等。具体的设计流程见图1。
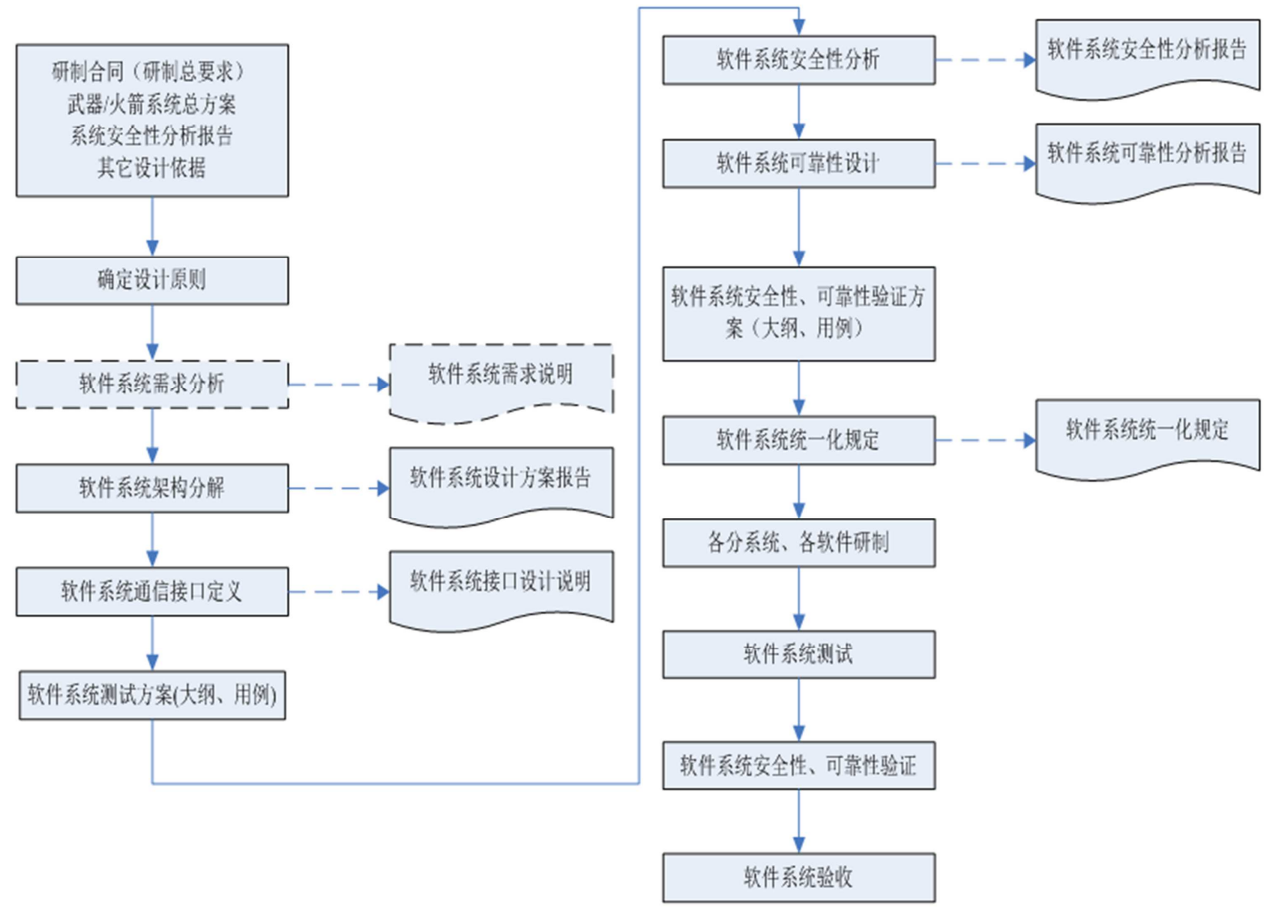

图1 软件系统设计流程图。 


\section{2. 软件研制过程的精细化管理}

《军用软件研制能力成熟度模型》（GJB5000A）借 用了美国卡内基-梅隆大学的软件工程研究所 (SEI) 为美 国国防部研究制定的一种评估软件承包商能力的办法, 强 调通过“过程管理”能力的提高, 最终实现软件研制质量由 组织的过程管理而非研发团队的能力来决定。

在研制、管理难度大的背景下, 导航系统软件研制工 作将GJB5000A理论与项目实践相结合, 按照GJB5000A二 级的 7 个 “过程域”的要求, 将软件研制过程细分为配置管 理、测量与分析、项目策划、项目监控、过程和产品质量 保证、需求管理和供方协议管理, 并分别制定了管理要求
根据项目策划过程的要求，对软件研制的工作量、进 度进行了估计, 制定了详细的进度表及风险管理计划、利 益相关方参与计划、资源管理计划、配置管理计划等, 并 对需重点决策的事件进行评估，规范了软件管理过程。同 时通过定期的测量及监控及时发现软件项目进展过程中 的问题, 及时解决, 避免问题在研制后期的集中爆发, 影 响软件研制进度及质量。

同时为了减少管理成本, 利于软件研制过程精细化管 理的深入推进, 在所级软件过程改进体系层面对依据软件 项目的具体情况制定分级管理原则, 执行不同的管理和技 术流程, 具体分级及对应的运行说明如表 1 所示:

表1 软件运行分级管理要求。

\begin{tabular}{|c|c|c|}
\hline 软件项目分级 & 软件项目运行说明 & 备注 \\
\hline 1级 & \multirow{4}{*}{ 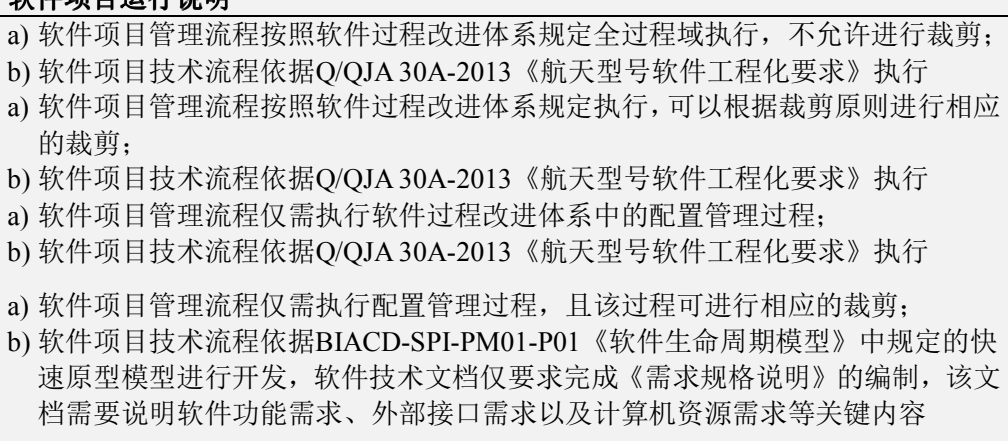 } & $\begin{array}{l}\text { 新研战略武器、宇航型号弹 (箭) 上软 } \\
\text { 件均须按照 } 1 \text { 级软件项目运行 }\end{array}$ \\
\hline 2级 & & $\begin{array}{l}\text { 处于运行过程中的或运维状态的战略武 } \\
\text { 器、宇航型号弹 (箭) 上软件按 } 2 \text { 级软件 } \\
\text { 项目运行 }\end{array}$ \\
\hline 3级 & & 战术武器配套软件 \\
\hline 4级 & & 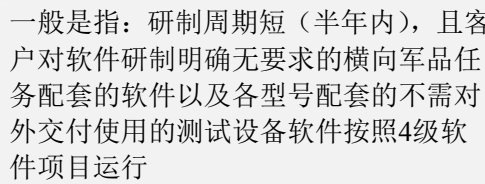 \\
\hline
\end{tabular}

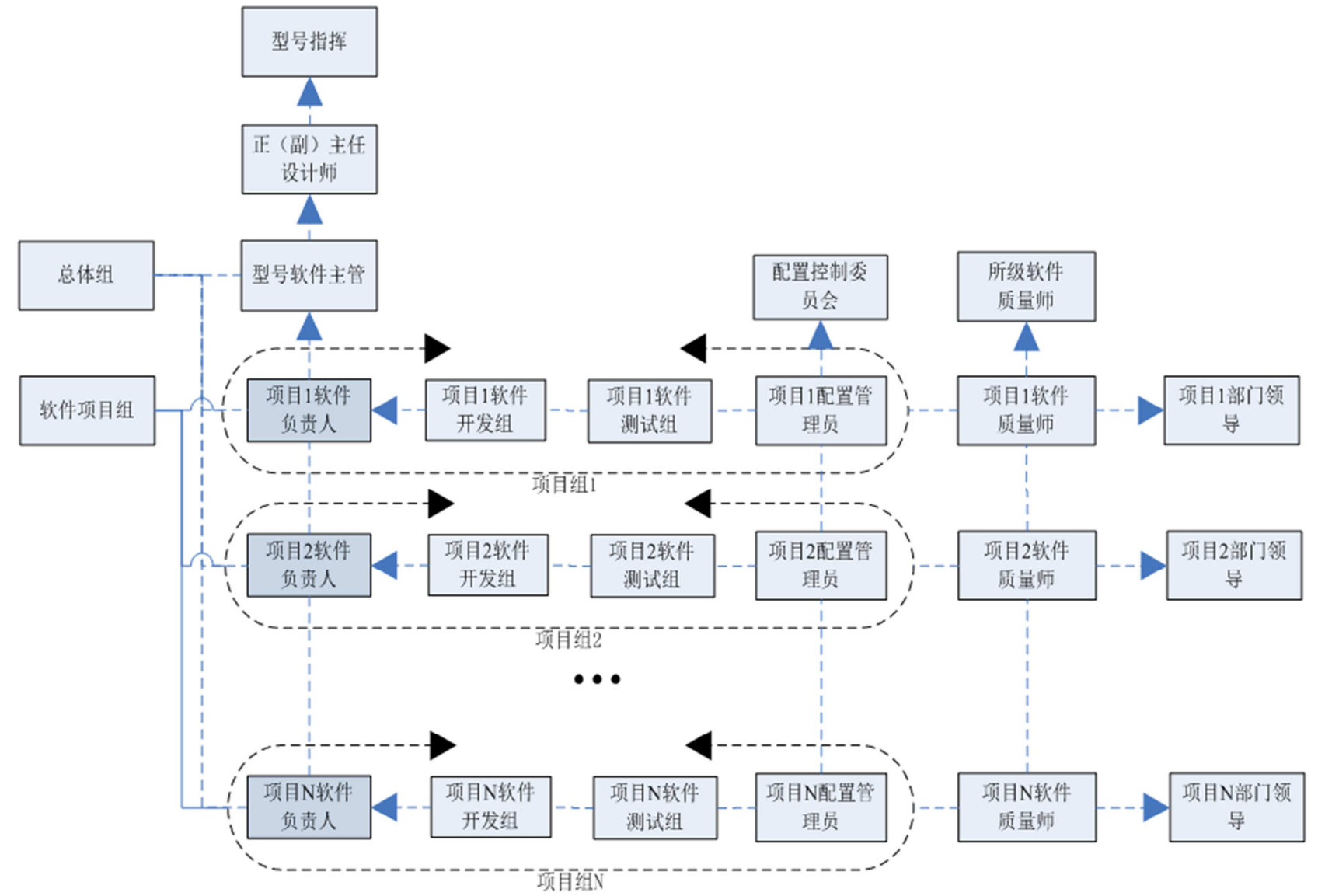

图2 跨部门型号软件研制管理模式示意图。 


\section{3. 建立良好的沟通及管理机制}

精密导航系统软件需要十余个软件配置项协同工作, 涉及多个软件研制部门及单位, 在这些软件研制部门及人 员中建立良好的沟通机制并对软件项目进行有效的管理 是需要重点关注的问题。首先, 导航系统软件多方协同的 核心在于软件接口界面。为保证风险可控, 在任务分解的 基础上, 首先明确多方软件的功能和职责, 进而划定软件 接口的数据流向, 责任控制主体, 确保接口定义清晰严密, 并形成正式的技术文件, 纳入配置管理库管理, 仅型号软 件主管具有修订的权利，防止多方修订造成的接口定义错 误, 当接口修订后及时入库并将新版本向相关人员进行发 布，保证软件更改及研制的顺利并实施。

另外结合单位软件研制实际, 针对系统软件研制跨部 门的情况建立了软件项目的管理新模式, 从横纵两条线分 别抓软件研制中的技术和管理, 并通过例会制度加强型号 主任设计师与各部门领导的有效沟通, 职责明确, 管理有 效, 具体见图1。其中型号主任设计师和型号软件主管负 责型号软件整体技术方案的制定和执行, 而涉及软件研制 的部门领导负责对项目进行管理并考核, 同时在承担软件 研制部门内部设置配置管理员和软件质量师分别负责项 目级配置管理和质量保证工作。

\section{4. 使用增量开发与传统瀑布模型结合的开发模式}

由于型号整体研制进度紧张, 为保证在需求变化, 尤 其是部分技术状态不确定情形下的软件质量控制, 导航系 统软件采用增量开发与传统瀑布模型结合的方式, 在整机 构建初期便提供先期验证版本以便支持整机快速测试验 证，同时也验证了软件方案的正确性。

在定义了系统需求后, 依据传统瀑布模型进行第一轮 的软件需求分析、总体架构设计, 详细设计及代码实现等 工作并形成一个可发布的版本, 后续随着功能的逐步完善 即采用序列化的创建产品的方法进行软件开发。每一个线 性序列产生软件的一个可发布的“增量”, 第一个建立的增 量完成预计功能/性能的一部分 (往往包含了核心功能, 即实现了基本的需求), 下一个增量实现另外的部分, 增 加更多的功能/性能, 然后与前面实现的增量进行集成, 如此循环, 直到系统完全实现。每个增量均须按软件工程 化要求进行相关文档的更新与评审及测试验证工作, 保证 增量实现的正确性及需求的可追踪性。

同时, 为了保证在此类开发模式下技术状态的可控及 可追溯性, 从更改有效性、影响分析完整性、试验验证充 分性及更改落实全面性进行技术状态控制。每个增量发布 后均需进行软件产品的入库工作, 以形成确定的软件技术 状态基线。具体流程见图2。

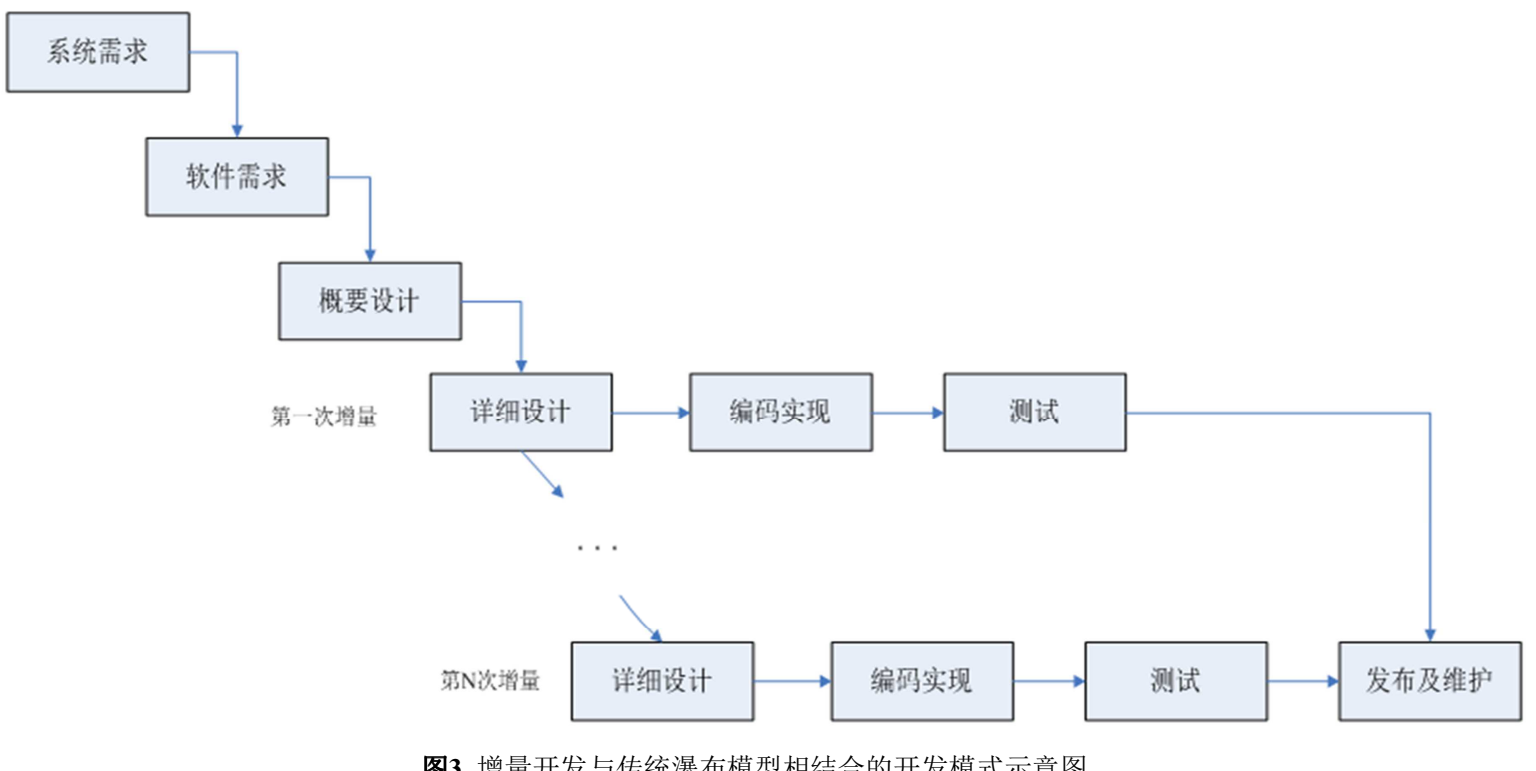

\section{5. 将可靠性、安全性设计贯穿软件研制整个生命周期}

精密导航系体软件具有安全关键等级高的特点, 在信 息流复杂, 进度紧张的前提下, 任何疏忽都可能对研制工 作造成颠覆性影响, 规范产品的可靠性、安全性设计、分 析、试验和验证过程成为重中之重且应贯穿软件研制整个 生命周期。

在系统需求分析阶段, 应提出软件可靠性要求并开展 软件危险分析; 在软件需求分析阶段应开展软件需求的可 靠性和安全性分析, 在软件设计阶段和代码实现阶段完成
软件可靠性、安全性的设计与验证工作。当面临下一轮的 增量开发时, 应对原有的可靠性及安全性分析结果进行梳 理, 确认是否有新增或不适用的可靠性及安全性措施。同 时采用独立评估的方式组织专家对软件功能、性能、接口、 中断等进行审查, 以形成准确的技术状态基线。

\section{4. 启示}

通过研制过程的合理管控, 导航系统软件在各研制阶 段技术状态严格受控, 并按计划交付。在各阶段测试及试 
验过程中, 软件各项指标均满足指标要求, 软件出厂后无 质量问题发生。导航系统软件研制工作管理实践为后续型 号软件研发积累了经验, 提供了借鉴, 并得到如下启示。

\section{1. 以 GJB5000A理论指导过程管理}

过程管理是保证软件产品质量稳定的重要手段, 并在 导航系统软件研制中起到了重要作用, 在研制过程中始终 坚持以GJB5000A来进行项目管理, 确定软件管理的的理 论基础, 确保软件研制的各个方面得到有效的管理, 指导 资源分配和保证项目进展状态得到有效控制, 才能最终保 证软件产品研制的效率和质量。

通过对研制过程的合理管控, 导航系统软件在各研制 阶段技术状态严格受控，问题及不符合项闭环率 $100 \%$ 、 计划节点完成率 $100 \%$, 在各阶段测试及试验过程中, 软 件可靠性指标、数据存储余量、总线负载余量、处理器机 时裕度均满足指标要求, 在型号软件专项复查中未发生技 术隐患, 软件出厂后无质量问题发生。

\section{2. 嵌入式软件开发方法优化}

\subsection{1. 嵌入式硬件构件开发方法}

导航系统软件中的嵌入式软件开发方法以结构化的 开发方法为主, 具有较高的执行效率, 但可扩展性和重用 性不高, 而嵌入式系统是软硬件紧密结合的系统, 所有的 软件都必须运行在特定的硬件体系上。因此有必要对面向 硬件模块的嵌入式系统开发方法进行研究, 即嵌入式硬件 构件的开发方法和基于硬件的底层软件的开发方法, 以提 高硬件系统的开发效率、可靠性及重用性, 便于系统维护。

硬件构件实现过程先将具体的功能模块按照硬件构 件的模型（包括功能模块、接口描述和模块描述）设计成 相应的硬件构件, 再将封装好的硬件构件添加到硬件构件 库中。接口是硬件构件与外部的通信渠道, 是连接两个硬 件构件的桥梁, 包括信号输入接口和信号输出接口。接口 由接口标识和接口对应实体组成, 接口对应实体就是接口 对应的硬件构件。当具体使用硬件构件时, 设计者只要通 过阅读硬件构件的模块描述, 了解构件的功能, 再根据实 际需求修改接口, 即可实现硬件构件的应用。

\subsection{2. 基于模型的嵌入式软件开发方法}

目前的航天型号软件开发模式还是一个典型的基于 代码的开发流程, 整个软件生命周期的核心是软件编码, 该开发方式存在的不足表现在: 文档以自然语言描述, 存 在歧义且无法测试; 手工编写的代码必须进行单元测试; 无法对需求进行验证。

采用以建模为核心的软件开发技术进行型号软件设 计, 将软件开发重点由编码阶段提前到设计阶段, 可以显
著提高软件的安全性和可靠性。同时, 可以通过模型仿真 的方式进行需求验证, 使得验证工作更加充分。

\section{5. 结论}

精密导航系统软件随整机交付后，经历了控制系统 综合匹配试验、总装出厂试验等试验验证, 技术受控, 各 项在轨验证与技术试验均显示了导航系统软件功能及性 能满足需求, 可靠性、安全性措施充分有效, 这些都充分 说明了导航系统软件质量保证充分, 过程管理有效。同时 精密导航系统软件研制工作的管理实践为后续型号软件 的研制奠定了技术与组织管理等方面的坚实基础。

\section{参考文献}

[1] 张晓龙.现代软件工程 $[\mathrm{M}]$. 北京: 清华大学出版社, 2011。

[2] 张海籓.软件工程导论 $[\mathrm{M}]$. 北京: 清华大学出版社, 2005。

[3] 金晶.对软件工程技术今后发展的思索[J].中国管理信息化, 2015, 18 (21)：162。

[4] 师迎海, 何雪慧. 迭代式软件开发模型研究及应用[J].微处理 机, 2015 (1)：55-57。

[5] 中国人民解放军总装备部.GJB 5000A-2008 军用软件研制 能力成熟度模型[S].北京: 总装备部军标出版发行部.2008。

[6] 陈涛, 李琦, 王金金, 等.宇航型号研制流程优化管理实践 [J]. 航天工业管理，2013（9）：64-67。

[7] 范洪涛,敏捷软件开发应用研究[D].成都: 西南交通大学, 2009。

[8] 林傘瀚.敏捷方法与小型团队的软件开发[J].软件导刊, 2014 (09)。

[9] 袁家军.航天工程精细化质量管理[J].中国工程科学, 2011, 13 (8) : 38-44。

[10] 怯肇乾.嵌入式系统硬件体系设计[M].北京: 北京航空航天 大学出版社, 2007。

[11] 刘杰.基于模型的设计及其嵌入式实现[M].北京: 北京航空 航天出版社, 2010。

[12] 王黎明.控制系统基于模型的系统工程开发方法研究[D].西 安: 西安电子科技大学, 2013。 